\title{
A Prospective Study to Evaluate Effectiveness of a Tinnitus Relief Device in a Clinical Setup
}

\author{
Nitin Aggarwal ${ }^{1}$ Soniya Gupta ${ }^{2}$ Prashant Goyal ${ }^{2}$ \\ ${ }^{1}$ Medicity ENT \& Vertigo Clinic, Noida, Uttar Pradesh, India \\ 2 Innoflaps Remedy Pvt. Ltd., Delhi, India
}

\begin{abstract}
Address for correspondence Prashant Goyal, B. Tech (Electronics and Communications), Innoflaps Remedy Pvt. Ltd., 99, New Rajdhani Enclave, Preet Vihar, Delhi 110092, India (e-mail: prashant@innoflaps.com).
\end{abstract}

\begin{abstract}
Keywords

- Tinnitus Relief device

- tinnitus

- Tinnitus Handicap Inventory

- effectiveness
\end{abstract}

Introduction Tinnitus has been shown to decrease the quality of life of a person. Being a less understood domain of the medical field, there are no definitive established therapies that can be applied to all patients of tinnitus. The current study is an effort to evaluate the effectiveness of the Tinnitus Relief device in the patients of tinnitus.

Aim To evaluate the effectiveness of Tinnitus Relief device TRx in treatment of tinnitus.

Methods A prospective study involving 67 participants suffering from tinnitus was conducted in a clinical setup at Delhi, Chandigarh, and Mumbai. These participants were subjected to pure tone audiometry (PTA) and an interview using Tinnitus Handicap Inventory (THI) questionnaire. These participants were advised to use Tinnitus Relief device TR201, TR301, TR401 for more than 6 to 10 months. Improvement in tinnitus in terms of percentage, questions pertaining to $\mathrm{THI}$, and overall improvement in life (subjective) were evaluated after discontinuation of the use of the device in each participant.

Results It was found that there was significant improvement in mean THI score, following treatment with Tinnitus Relief TRx. This device was also able to completely alleviate 5 out of 12 associated complaints of tinnitus. No association could be found between the use of Tinnitus Relief therapy and hearing loss.

Conclusion Tinnitus Relief TRx can emerge as an effective tool for treatment of the tinnitus and can improve the life of the sufferer.

\section{Introduction}

Tinnitus or has been one of the less explored conditions in the field of medicine. It is defined as the conscious perception of sound in absence of external stimulus. This condition can be classified as primary or secondary. The exact causation of the primary (or subjective) tinnitus has not been identified. However, literature suggests that tinnitus is most commonly observed among patients of hearing loss. ${ }^{1}$ One of the major risk factors responsible for tinnitus is "exposure to loud

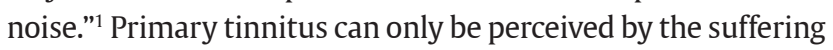
patient. It cannot be detected by the examiner with or without amplification. However, changes have been observed in the electroencephalography (EEG) or magnetoencephalography signals from the patients of primary tinnitus, which mostly indicates involvement of temporal lobe of the brain. ${ }^{2}$ It has been believed that, it is the absence of auditory stimulus in hearing loss, which triggers altered sensations resulting in tinnitus. The secondary tinnitus has been linked to various organic conditions pertaining to anatomical structure of middle ear or blood vessel. Essentially, the sound of secondary tinnitus can be perceived by the examiner, with or without amplification. Tinnitus has been described as number of expressions such as wheezing, humming, ticking, and sound of a cricket. 
Data on the exact burden of tinnitus are not available in India. Various studies conducted in western countries have revealed prevalence of tinnitus as between $8 \%$ and $15 \% .{ }^{3}$ Some studies have also indicated its prevalence as high as $25 \% .{ }^{4}$ Although the incidence of tinnitus varies within different age groups, there are clear evidences available which indicate a rise in the incidence of tinnitus with presbycusis and aging. ${ }^{5}$ When the burden is estimated in sheer number of persons suffering from the tinnitus, it reaches to 40 to 50 million people worldwide. Approximately 5\% of them experience severe debilitating effect of tinnitus. ${ }^{6}$

The tinnitus has been known to decrease the quality of life by causing stress, annoyance, anxiety, and disturbances in the sleep. The degree of its effect on the life of a patient depends on the severity. There have been cases where tinnitus has led to depression and ultimately suicidal tendencies. ${ }^{7}$

The instrumental quantification is almost impossible in cases of primary tinnitus. Even in cases of secondary tinnitus, the mental or psychological effects cannot be measured by the conventional available instruments. For the same, tools such as Tinnitus Handicap Inventory (THI) have been designed to measure the level of handicap imparted by the tinnitus. ${ }^{8}$

At this juncture, according to the cause and severity of the tinnitus, multiple therapies are available and several of them are still being evaluated for effectiveness. It can be safely said that treatment of tinnitus is still an enigma for medical field. The large basket of treatment modalities includes auditory stimulation with sound enrichment, implantable devices, behavior therapy, pharmacological therapy, tinnitus retraining therapy, surgical correction of structural abnormalities of blood vessels, neuromodulation, counseling, and many more which are under research. However, the effectiveness of these treatments has shown variations in different research studies. None of them can be labeled as a definitive treatment for primary tinnitus. The sound therapy or masking has failed to show enough evidence of its effectiveness in treatment of tinnitus. ${ }^{9}$ A study conducted by Ahmed and Khater have shown cochlear implant as an effective method to suppress tinnitus. ${ }^{10}$ Cognitive behavioral therapy has shown positive effects on alleviating annoyance caused by tinnitus, but it has not been effective in suppression of tinnitus. ${ }^{11}$ Pharmacotherapy and surgical corrections have been effective in secondary tinnitus, but they have not revealed their role in suppressing primary tinnitus. Tinnitus retraining therapy (TRT) is a combination of counseling and sound therapy. Previous studies have demonstrated that TRT has been effective in reducing the interference of tinnitus in patients' life. However, it could remove tinnitus completely in approximately $20 \%$ of the patients. ${ }^{12}$

Current study was based on a newly developed Tinnitus Relief TRx, which can be a potential tool in management of the tinnitus. It is a noninvasive device, which requires minimal training for effective use. Being easy and handy device, it is suitable for patients of all ages and educational classes. However, before applying the device in clinical management, there is requirement of research to evaluate its effectiveness.
Present study aimed to evaluate and quantify the effectiveness of Tinnitus Relief TRx in treatment of tinnitus.

\section{Methods}

Present study was a prospective study among patients diagnosed with tinnitus. The study was conducted from 10 June 2017 to 8 April 2019. DrSpectra clinic is a tertiary level health care center, located at Delhi, Chandigarh, and Mumbai. A purposive sampling method was adopted for selection of a cohort of 67 patients suffering from tinnitus. At the time of enrolment, interviews were conducted to collect baseline information using a prestructured questionnaire. The questionnaire included sociodemographic information, information regarding associated problems, and questions to calculate THI. They were also subjected to Pure tone audiometry (PTA) for both ears. These patients were advised to use Tinnitus Relief TRx for more than 6 months. However, the actual use of the device by patients varied. After the discontinuation of the use of device, each of these patients was interviewed. At the time of follow-up interview, they were asked about the improvement in tinnitus in terms of percentage, questions pertaining to THI, and overall improvement in life (subjective). Four of the study participants were subjected to follow-up PTA as per the indication.

An informed consent was ensured from each patient before enrolment into the study.

Inclusion criteria:

- This study is for primary tinnitus (or subjective).

- Male and female patients with age 18 years or more.

- Diagnosed with tinnitus (both recent onset and persistent).

- Without any long history of clinical psychiatric illness before tinnitus started or history of treatment with/ consumption of psychotropic drugs.

\section{Exclusion criteria}

- Patients with profound hearing loss

- Patients with history or evidence of significant brain malformation or neoplasm, head injury, cerebral vascular events, seizure, and neurodegenerative disorder affecting the brain or prior brain surgery.

\section{Tinnitus Relief Device}

Tinnitus Relief Device (Innoflaps Remedy Pvt Ltd, Delhi, India) is a small medical device (a tinnitus relief device) that looks like a mobile and coupled via comfortable, high-quality medical earphones. The device delivers a pattern of acoustic stimuli designed to retrain the neural pathways and is customized for your hearing and tinnitus. A treatment program that includes ongoing personalized support, education, and monitoring from an expert who specializes in tinnitus.

\section{Ethical Clearance}

Current study was conducted by individuals, in guidance of innovators and senior doctors. The Tinnitus Relief device, 
Table 1 Tinnitus severity category

\begin{tabular}{|l|l|}
\hline THI score & Category \\
\hline $0-16$ & Slight or no handicap (Grade 1) \\
\hline $18-36$ & Mild handicap (Grade 2) \\
\hline $38-56$ & Moderate handicap (Grade 3) \\
\hline $58-76$ & Severe handicap (Grade 4) \\
\hline $78-100$ & Catastrophic handicap (Grade 5) \\
\hline
\end{tabular}

used for this study is developed by Innoflaps remedy Pvt. Ltd. (Clinic Name is Drspectra) and it has been filed for patent, which is under review. It is a class IIA device and the other study is also conducted by institution. This device is used for external use which is safe for humans.

Tinnitus Handicap Inventory $(\mathrm{THI})^{8}$ : $\mathrm{THI}$ is a questionnaire to identify and quantify handicap experienced by the patient because of tinnitus. It consists of 25 questions, for which the responses are recorded using the Likert scale. Each response is given appropriate score and a total is obtained at the end of questionnaire. Based on this total, the person was categorized as below ( - Table $\mathbf{1}$ ).

\section{Statistical Analysis}

The data were analyzed using the SPSS 25 software (Reseapro Scientific Services (P) Ltd., Bhubaneswar, India). The results were expressed in frequencies, percentage, mean, and standard deviation (SD). Paired $t$-test and Chi square test were applied appropriately to assess the association between variables.

\section{Results}

The study included 47 (70.1\%) females and 20 (29.9\%) males (-Table 2). It was found in PTA that the mean hearing loss among study participants was almost similar in both ears (with $35.4 \mathrm{~dB}[\mathrm{~dB}]$ in right ear and $36.5 \mathrm{~dB}$ in left ear). Mean age of the study sample was 40.6 years with standard deviation of 17.1 years. The majority of the study sample was constituted by the age group of 16 to 30 years (32.8\%), followed by age group of 31 to 45 years (28.4\%). The median duration of the tinnitus among study participants was 8 months. Majority of the study participants were suffering from tinnitus for 7 to 12 months (31.3\%). Majority (95.5\%) of the participants were mild to catastrophic handicap according to THI. Out of them most of them were classified as severe handicap (34.3\%).

Tinnitus was described with various terms by the study participants ( - Fig. 1). For example, maximum participants (31) described tinnitus as "whistling" sound. Least number of participants (2) described it as a "sound of blowing air." Many of them described tinnitus with even more than one term.

The mean baseline THI score was found to be 57.2 with standard deviation of 21.6. After using Tinnitus Relief TRx, the mean THI score had decreased to 30.9 with standard deviation of 20.7. Paired $t$-test was applied to assess the difference between these two values and the difference was found to be significant $(p<0.05)$. So, it can be deduced from this result
Table 2 Characteristics of the study sample at the time of enrolment

\begin{tabular}{|c|c|}
\hline Characteristics & Value \\
\hline \multicolumn{2}{|l|}{ Gender } \\
\hline Male (number/percentage) & $20(29.9 \%)$ \\
\hline Female (number/percentage) & $47(70.1 \%)$ \\
\hline \multicolumn{2}{|l|}{ Hearing loss (in decibels) } \\
\hline PTA-Right ear (mean \pm SD) & $35.4 \pm 22.2$ \\
\hline PTA-Left Ear (mean \pm SD) & $36.5 \pm 22.0$ \\
\hline \multicolumn{2}{|l|}{ Age distribution (in completed years) } \\
\hline Less than 16 & $2(3.0 \%)$ \\
\hline $16-30$ & $22(32.8 \%)$ \\
\hline $31-45$ & $19(28.4 \%)$ \\
\hline $46-60$ & $16(23.9 \%)$ \\
\hline More than 60 & $8(11.9 \%)$ \\
\hline Mean age (mean \pm SD) in completed years & $40.6 \pm 17.1$ \\
\hline \multicolumn{2}{|l|}{ Duration of tinnitus (in completed months) } \\
\hline$\leq 6$ & $14(20.9 \%)$ \\
\hline $7-12$ & $21(31.3 \%)$ \\
\hline $13-18$ & $8(11.9 \%)$ \\
\hline $19-24$ & $9(13.4 \%)$ \\
\hline$\geq 25$ & $12(17.9 \%)$ \\
\hline \multicolumn{2}{|l|}{ THI categories } \\
\hline Slight or no handicap & $3(4.5)$ \\
\hline Mild handicap & $11(16.4)$ \\
\hline Moderate handicap & $17(25.4)$ \\
\hline Severe handicap & $23(34.3)$ \\
\hline Catastrophic handicap & $13(19.4)$ \\
\hline
\end{tabular}

"The duration of tinnitus was unknown among 3 patients.

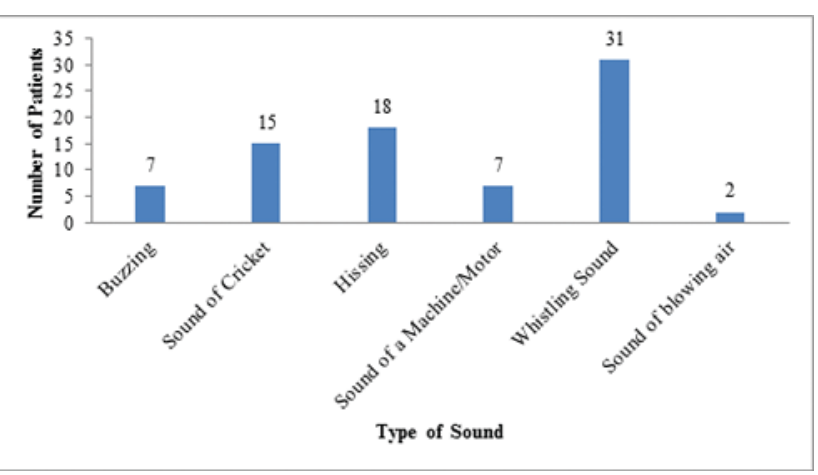

Fig. 1 Types of tinnitus experienced by the study participants before treatment $(N=67)$.

that treatment with Tinnitus Relief TRx significantly reduces the level of handicap amongst patients of tinnitus ( - Table 3 ).

Further analysis was done to assess the effect of duration of treatment on the improvement in THI scores. The findings of the same are in - Table 4 .

During data analysis, the study group was divided, based on the duration of treatment, into two groups-those who 
Table 3 THI scores before and after treatment

\begin{tabular}{|l|l|l|}
\hline THI score & Mean \pm SD & p-Value* \\
\hline Before treatment & $57.2 \pm 21.6$ & $<0.05$ (significant) \\
\hline After treatment & $30.9 \pm 20.7$ & \\
\hline
\end{tabular}

* Paired $t$-test.

Table 4 Improvement in hearing among patients (after treatment)

\begin{tabular}{|l|l|l|l|}
\hline & $\begin{array}{l}\text { PTA before } \\
\text { treatment }(\mathrm{dB}) \\
(\text { mean } \pm \text { SD) }\end{array}$ & $\begin{array}{l}\text { PTA after } \\
\text { treatment }(\mathrm{dB}) \\
(\text { mean } \pm \text { SD) }\end{array}$ & $p$-Value* \\
\hline Right ear & $48.44 \pm 37.42$ & $39.24 \pm 33.08$ & 0.40847 \\
\hline Left ear & $37.96 \pm 19.44$ & $25.98 \pm 13.49$ & 0.24825 \\
\hline
\end{tabular}

*Paired $t$-test.

received treatment for 2 months or less and those who received treatment for more than 2 months. The mean THI score of both the groups were explored before and after treatment and comparisons were done. It was found that there was significant improvement in THI scores in both the groups.

Four patients were subjected to PTA after the end of treatment according to indications (-Table 5). Although improvement in the hearing was observed in both ears, statistically significant difference could not be found.

The mean of subjective (percentage) improvement in tinnitus were compared between two groups defined based on the treatment duration (-Table 6). The mean improvement in tinnitus was approximately $60 \%$ among the first group of those who received treatment for 2 months or less. The mean improvement in tinnitus was approximately 64\% among the second group of those who received treatment for more than 2 months. No statistical difference could be found between these two values. Similarly, the mean difference of THI scores between pre- and posttreatment was found. It was approximately 26.2 in the first group and 26.4 in the second group. Again, no statistical difference could be found between these two values.

As evident from the $\mathbf{- T a b l e ~} \mathbf{7}$, the most common problem associated with tinnitus was irritation (76.1\%) followed by heaviness in the head (55.2\%). After treatment, 100\% improvement was observed in the complaints of heaviness in ears, difficulty in concentration, vertigo, anger, and depression.

As evident from - Fig. 2, there was improvement in terms of severity of handicap categories after treatment with Tinnitus Relief TRx. Maximum improvement (24.9\%) was observed among patients who were severely handicap before treatment, followed by those who were catastrophic handicap (17.9\%).

Table 7 Comparison of problems associated with tinnitus before and after treatment

\begin{tabular}{|l|l|l|}
\hline Problem & $\begin{array}{l}\text { Before treatment } \\
\text { frequency (\%) }\end{array}$ & $\begin{array}{l}\text { After treatment } \\
\text { frequency (\%) }\end{array}$ \\
\hline Anxiety & $3(4.5)$ & $1(1.4)$ \\
\hline $\begin{array}{l}\text { Difficulty in } \\
\text { concentration }\end{array}$ & $24(35.8)$ & $0(0.0)$ \\
\hline $\begin{array}{l}\text { Heaviness in } \\
\text { head }\end{array}$ & $37(55.2)$ & $3(4.5)$ \\
\hline $\begin{array}{l}\text { Heaviness in } \\
\text { the ear }\end{array}$ & $7(10.4)$ & $0(0.0)$ \\
\hline $\begin{array}{l}\text { Difficulty in } \\
\text { hearing }\end{array}$ & $20(29.9)$ & $5(7.5)$ \\
\hline Hyperacusis & $6(9.0)$ & $16(23.9)$ \\
\hline Irritation & $51(76.1)$ & $4(6.0)$ \\
\hline $\begin{array}{l}\text { Sleep } \\
\text { disturbances }\end{array}$ & $41(61.2)$ & $0(0.0)$ \\
\hline Vertigo & $4(6.0)$ & $2(3.0)$ \\
\hline Headache & $13(19.4)$ & $0(0.0)$ \\
\hline Anger & $1(1.4)$ & $0(0.0)$ \\
\hline Depression & $1(1.5)$ & \\
\hline
\end{tabular}

Table 5 Comparison of THI before treatment and after treatment in two groups of patients based on duration of treatment

\begin{tabular}{|l|l|l|l|l|}
\hline THI & Treatment received for $\leq 2$ months & $p$-Value & $\begin{array}{l}\text { Treatment received } \\
\text { for }>2 \text { months }\end{array}$ & $p$-Value \\
\cline { 1 - 4 } Before treatment & $58.82 \pm 19.469$ & $<0.05$ & $56.68 \pm 22.488$ & $<0.05$ \\
\cline { 1 - 2 } After treatment & $32.35 \pm 24.731$ & & $30.40 \pm 19.445$ & \\
\hline
\end{tabular}

* Paired t Test

Table 6 Comparison of subjective improvement (in percentage) and pre and post differences in two groups based on the treatment duration

\begin{tabular}{|l|l|l|l|}
\hline & $\begin{array}{l}\text { Treatment received } \\
\text { for } \leq 2 \text { months }\end{array}$ & $\begin{array}{l}\text { Treatment received } \\
\text { for }>2 \text { months }\end{array}$ & $\begin{array}{l}p \text {-Value } \\
\text { (paired } t \text {-test) }\end{array}$ \\
\hline Percentage improvement & $60.00 \pm 33.54$ & $64.1 \pm 26.35$ & 0.607 \\
\hline $\begin{array}{l}\text { Difference between THI scores of pretreatment and } \\
\text { post-treatment phase }\end{array}$ & $26.28 \pm 21.15$ & $26.47 \pm 19.07$ & 0.972 \\
\hline
\end{tabular}




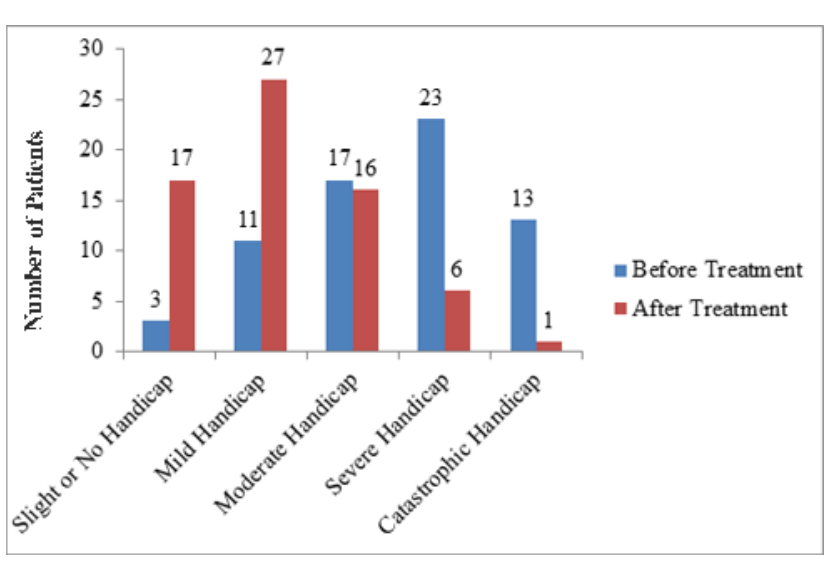

Fig. 2 Severity of tinnitus based on THI before and after treatment with Tinnitus relief device.

\section{Discussion}

Numerous researches have shown that tinnitus is mostly associated with high-frequency hearing loss (HL), which is seen in presbycusis. ${ }^{13}$ Current study showed that tinnitus was most commonly seen in the age group of 16 to 30 years (32.8\%), followed by the age group of 31 to 45 years $(28.4 \%)$. Current study did not have the adequate representation of geriatric population to validate this finding. The average hearing loss in present study was found to be around 35 to $36 \mathrm{~dB}$. This pattern is comparable with the findings of Langguth et al. ${ }^{14}$

In the present study, the most common type of tinnitus experienced by the study participants was "whistling" sound. An India-based research study conducted by Makar et al found "buzzing" as the most common type of tinnitus sound, followed by "whistling." 15 The mean HL in the study subject was approximately $35 \mathrm{~dB}$. However, the mean HL in the referred study was approximately $53 \mathrm{~dB}$. The mean THI score before treatment with Tinnitus Relief TRx was found to be 57.2 with standard deviation of 21 .6. In the study conducted to evaluate the effectiveness of sound therapy, the mean baseline THI was 55.8, which is comparable with the current study. In the current study mean duration of treatment was around 7 months. ${ }^{16}$ After treatment, the mean THI score was reduced to 30.9. This finding is comparable to the findings by Suzuki et al, who showed reduction of THI score to 29.6 after 6 months. However, the current study failed to show the effect of duration of treatment on THI score, as it is limited by single follow-up. ${ }^{16}$

Current study showed that pretreatment THI score of $57.2 \pm 21.6$ (mean \pm SD) decreased to $30.9 \pm 20.7$, which is improvement of almost 26.3 points or $46 \%$. In comparison, tinnitus retreatment therapy with narrow band sound showed decrease in THI score from $48.25 \pm 25.56$ to $42.00 \pm 29.55 .{ }^{17}$ So it can be seen that Tinnitus Relief TRx has better effect than TRT. In the same study significant improvement was seen in case of therapy with broadband sound (from $54.22 \pm 22.41$ to $35.11 \pm 22.44$ ). Similar results were also found by S. Goel and S. Gupta who could observe 56\% improvement in THI scores following treatment with Tinnitus Relief device. ${ }^{18}$
Another study conducted by Simonetti et al evaluated the effect of fractal tone on THI. ${ }^{19}$ They showed decrease in THI from $45.17 \pm 11.84$ to $25.60 \pm 4.77$, which is $\sim 43.3 \%$ improvement. Compared with that, in the similar duration current study demonstrated improvement of $46 \%$ in THI score. Neuromodulation is also a recognized treatment modality for tinnitus. A study conducted at the University Medical Center Groningen, the Netherlands, evaluated the effect of direct stimulation of cochlear nerve on tinnitus. ${ }^{20}$ They found that after 49 months, the reduction in THI was $24 \pm 26$. In comparison, Tinnitus Relief device has demonstrated mean decrease of 26.3 points in mean therapy duration of 7 months. Use of Tinnitus Relief device is a noninvasive method, which gives it an added advantage.

In the present study, no significant improvement in PTA was found with significant improvement in THI score. This suggests that there is no statistically significant effect of Tinnitus Relief TRx on the hearing of the patient. The present study also revealed that the Tinnitus Relief device was able to relieve 5 out of 12 problems which were associated with tinnitus before treatment. This effect is reflected in the improvement of quality of life in terms of improvement in THI scores.

Present study demonstrated improvement in the level of handicap (determined by THI scores) following Tinnitus Relief TRx. Similar improvement was also observed by S. Goel and S. Gupta. ${ }^{18}$ In a study conducted by Ho Yun Lee revealed that there was reduction of THI score of $\sim 28.69$ points following bifrontal transcranial direct current stimulation. $^{21}$

Recently new treatment options have emerged in form of repetitive transcranial magnetic stimulation and deep brain stimulation. However, most of them are still under investigation for their effect. Formanek et al found that there was no significant effect of repetitive transcranial magnetic stimulation on THI. ${ }^{21}$ Past research has demonstrated significant reduction in THI by deep brain stimulation, but being an experimental and invasive procedure, its effectiveness is under debate. ${ }^{22}$

\section{Limitations of the Study}

The sample size of the present study was small to generalize its findings. More research is required to confirm the findings of current study. The representation of geriatric population was inadequate in the present study. So, it may be biased to generalize the findings in the geriatric population.

\section{Conclusion}

Tinnitus Relief device (Innoflaps Remedy Pvt Ltd, Delhi, India) was found to be an effective tool to treat the tinnitus, especially in terms of level of handicap. When compared with other established and experimental therapies, Tinnitus Relief TRx was found to be more effective. This device was also effective in alleviating the complaints associated with tinnitus. All feedbacks of the patients are available on our YouTube channel (www.youtube.com/drspectra). 


\section{Conflict of Interest}

None declared.

\section{References}

1 Hauptmann C, Williams M, Vinciati F, Haller M. Technical feasibility of acoustic coordinated reset therapy for tinnitus delivered via hearing aids: a case study. Case Rep Otolaryngol 2017;2017:5304242

2 Eggermont JJ, Tass PA. Maladaptive neural synchrony in tinnitus: origin and restoration. Front Neurol 2015;6:29

3 Gudex C, Skellgaard PH, West T, Sørensen J. Effectiveness of a tinnitus management programme: a 2-year follow-up study. BMC Ear Nose Throat Disord 2009;9:6

4 Nondahl DM, Cruickshanks KJ, Wiley TL, Klein R, Klein BE, Tweed TS. Prevalence and 5-year incidence of tinnitus among older adults: the epidemiology of hearing loss study. J Am Acad Audiol 2002;13(6):323-331

5 Podoshin L, Ben-David J, Teszler CB. Pediatric and geriatric tinnitus. Int Tinnitus J 1997;3(2):101-103

6 Davis A, El Refaie A. Epidemiology of tinnitus. In: RS Tyler, ed. Tinnitus Handbook. San Diego, CA: Thomson Learning; 2000;1-23

7 Ziai K, Moshtaghi O, Mahboubi H, Djalilian HR. Tinnitus patients suffering from anxiety and depression: a review. Int Tinnitus J 2017;21(1):68-73

8 Newman CW, Jacobson GP, Spitzer JB. Development of the Tinnitus Handicap Inventory. Arch Otolaryngol Head Neck Surg 1996;122(2):143-148

9 Hobson J, Chisholm E, El Refaie A. Sound therapy (masking) in the management of tinnitus in adults. Cochrane Database Syst Rev 2012;11:CD006371

10 Ahmed MF, Khater A. Tinnitus suppression after cochlear implantation in patients with single-sided deafness. Egypt J Otolaryngol 2017;33:61-66

11 Jun HJ, Park MK. Cognitive behavioral therapy for tinnitus: evidence and efficacy. Korean J Audiol 2013;17(3):101-104
12 Jastreboff PJ, Jastreboff MM. Tinnitus Retraining Therapy (TRT) as a method for treatment of tinnitus and hyperacusis patients. J Am Acad Audiol 2000;11(3):162-177

13 Hesse G. Evidence and evidence gaps in tinnitus therapy. GMS Curr Top Otorhinolaryngol Head Neck Surg 2016;15:Doc04

14 Langguth B, Landgrebe M, Schlee W, et al. Different patterns of hearing loss among tinnitus patients : a latent class analysis of a large sample. Front Neurol 2017;8:46

15 Makar SK, Biswas A, Shatapathy P. The impact of tinnitus on sufferers in Indian population. Indian J. Otolaryngol Head Neck Surg 2014; 66(Suppl 1): 37-51

16 Barros Suzuki FA, Yonamine FK, Onishi ET, Penido NO, Oliveira $\mathrm{N}$. Effectiveness of sound therapy in patients with tinnitus resistant to previous treatments: importance of adjustments. Rev Bras Otorrinolaringol (Engl Ed) 2016;82(3):297-303

17 Kim BJ, Chung SW, Jung JY, Suh MW. Effect of different sounds on the treatment outcome of tinnitus retraining therapy. Clin Exp Otorhinolaryngol 2014;7(2):87-93

18 Goel S, Gupta S. Tinnitusrelief ${ }^{\mathrm{TM}}$ device in treatment of bothersome tinnitus : preliminary experience in private setting. Glob J Otolaryngol 2017;5:2-4

19 Simonetti P, Vasconcelos LG, Oiticica J. Effect of Fractal Tones on the Improvement of Tinnitus Handicap Inventory Functional Scores among Chronic Tinnitus Patients: An Open-label Pilot Study. Int Arch Otorhinolaryngol 2018;22(4):387-394

20 van den Berge MJ, van Dijk JM, Free RH, Stienstra J, van Dijk P, van der Laan BF. Effect of direct stimulation of the cochleovestibular nerve on tinnitus: a long-term follow-up study. World Neurosurg 2017;98:571-577

21 Formánek M, Migalová P, Krulová P, et al. Combined transcranial magnetic stimulation in the treatment of chronic tinnitus. Ann Clin Transl Neurol 2018;5(7):857-864

22 Smit JV, Janssen MLF, Engelhard $M$, et al. The impact of deep brain stimulation on tinnitus. Surg Neurol Int 2016;7(Suppl 35):S848-S854 